Advanced Computing: An International Journal (ACIJ), Vol.5, No.2/3, May 2014

\title{
Downlin K BeAMForming AND AdMission CONTROL FOR SPECTRUM SHARING COgNITIVE RADio Mimo SySTEM
}

\author{
A. Suban ${ }^{1}$, I. Ramanathan ${ }^{2}$ \\ ${ }^{1}$ Assistant Professor, Dept of ECE, VCET, Madurai, India \\ ${ }^{2}$ PG Student, Dept of ECE, VCET, Madurai, India
}

\begin{abstract}
We investigate the problem of multiuser downlink beam-forming and admission control considering a scenario where licensed, or primary, users and cognitive radios, or secondary users, are transmitting concurrently over the same band. The primary users share a common receiver and the interference on this receiver from secondary users should be strictly limited to a certain level. Each secondary link is assumed to have a minimum quality of service (QoS) requirement that should be satisfied together with the interference limit constraint, otherwise the secondary link is not admitted. Under those constraints, downlink beam-forming and admission control for secondary users are investigated for two main optimization objectives. First, we maximize the number of admitted secondary links. Second, we maximize the sum throughput of the admitted secondary links. our main design objective is to minimize the transmit power of the SU-transmitter while simultaneously targeting a lower-bound on the received signal-tointerference-plus-noise-ratio(SINR) for the SU's and imposing on upper limit on the interference power(IP) at the PUs.
\end{abstract}

\section{KEYWORDS}

Cognitive radios, downlink beam-forming and admission control, convex optimization.

\section{INTRODUCTION}

Recent studies by the FCC show that the current utilization of some spectrum bands is as low as $15 \%$ [1]. On the other hand, it is commonly believed that there is a crisis of spectrum availability at frequencies that can be economically used for wireless communications via fixed spectrum allocation. In other words, there is fierce competition for the use of spectra, especially in the bands below $3 \mathrm{GHz}$ while in the same time most of these bands suffer from underutilization. Therefore, there is an increasing interest in developing new efficient techniques for spectrum management and sharing as in [2] which consequently motivates the concept of cognitive radios [3] and dynamic spectrum access.

In this paper we consider the problem of admission and power control for cognitive radios in a spectrum underlay network where cognitive or secondary users share the same spectrum band concurrently with the licensed or primary users under the following constraint. The interference caused by the secondary users on the primary network has to be kept below a maximum 
allowable limit. We impose an additional constraint that each secondary user has a minimum quality of service (QoS) requirement that should be satisfied.

A lot of work addresses power and admission control for cognitive radios in underlay networks under similar constraints. In [4], a centralized algorithm based on tree-pruning technique was proposed, the proposed algorithm leads to the largest supported set of secondary links but with extensive computations. Moreover, a distributed game theoretic approach based on sequential play was introduced, but it converges to locally optimal solutions. In [5], a distributed algorithm was introduced that aims at minimizing the total transmit power by primary and secondary links. However, the primary users were allowed to increase their transmission power without bounds.

In order to maximize the number of admitted secondary links under QoS and interference constraints, the authors of [6] developed two centralized less complicated, but suboptimal, algorithms namely I-SMIRA and I-SMART(R). The two algorithms are based on permanent removals of links. In [7], joint admission control and rate/power allocation for secondary links was investigated while only mean channel gains are available. The main objective was to admit secondary users so that the sum throughput is maximized under two fairness criteria, namely, proportional fairness and max-min fairness. However, the removal technique used does not necessarily maximize the number of admitted secondary links. In [8], power control for both primary and secondary users is considered for the sake of maximizing the total throughput of both the primary and secondary networks. The QoS of each primary link, however, is guaranteed to be maintained above a minimum limit.

In this paper, our main objective is to maximize the sum throughput for the maximum number of secondary links that can be admitted to the network under the fore mentioned constraints. In order to render this task tractable, we divide it into two sub-problems. The first is the maximization of the number of admitted secondary links under the aforementioned constraints, whereas the second is to maximize the sum throughput of the admitted secondary links.

The first sub-problem becomes NP-hard when not all secondary users can be supported at their QoS requirements, and the second sub-problem is non-convex. An algorithm based on monotonic optimization has been introduced and proven to achieve the global optimal solution of sum throughput maximization. However, its computations are still rather extensive as the algorithm searches for the optimal solution over the boundary of the feasible set. Moreover, the problem of sum rate maximization in wireless networks resembles the corresponding problem in DSL. There has been considerable research on approximation algorithms for this problem as in [9].

The main contributions of this paper are:

- We consider maximizing the sum throughput for the set of admitted secondary links. The optimal solution to this problem is computationally prohibitive because the problem is nonconvex. However, we introduce an iterative algorithm based on geometric programming (GP) and prove the convergence to, at least, a local optimum solution. The proposed iterative algorithm requires simpler computations than the single condensation methods proposed, and the double condensation method described without a proof of its convergence.

- We show, via simulations, the trade-off between the number of admitted secondary links and the sum throughput of the secondary network. In general, maximizing the sum throughput of the secondary network with guarantees on the QoS of every admitted link results in less 
throughput than maximizing the sum throughput without those guarantees. However, we show numerically, that the difference in throughput between the two cases is not significant.

The rest of this paper is organized as follows. In Section II, the system model is introduced, and the problem is formulated. In Section III, an algorithm for maximizing the number of admitted secondary links under the QoS and interference constraints is proposed. In Section IV, maximizing the sum throughput of the admitted secondary links is addressed and an iterative algorithm is developed. Simulation results are presented in Section V, and the paper is concluded in Section VI.

\section{COGNITIVE RADIO AND PROBLEM FORMULATION}

Cognitive radio technology is the key technology that enables an underlay network to use spectrum in a dynamic manner. The term, cognitive radio, can formally be defined as follows A "Cognitive Radio" is a radio that can change its transmitter parameters based on interaction with the environment in which it operates.

From this definition, two main characteristics of the cognitive radio can be defined:

- Cognitive capability: Cognitive capability refers to the ability of the radio technology to capture or sense the information from its radio environment. This capability cannot simply be realized by monitoring the power in some frequency band of interest but more sophisticated techniques are required in order to capture the temporal and spatial variations in the radio environment and avoid interference to other users. Through this capability, the portions of the spectrum that are unused at a specific time or location can be identified. Consequently, the best spectrum and appropriate operating parameters can be selected.

- Reconfigurability: The cognitive capability provides spectrum awareness whereas reconfigurability enables the radio to be dynamically programmed according to the radio environment. More specifically, the cognitive radio can be programmed to transmit and receive on a variety of frequencies and to use different transmission access technologies supported by its hardware design. 


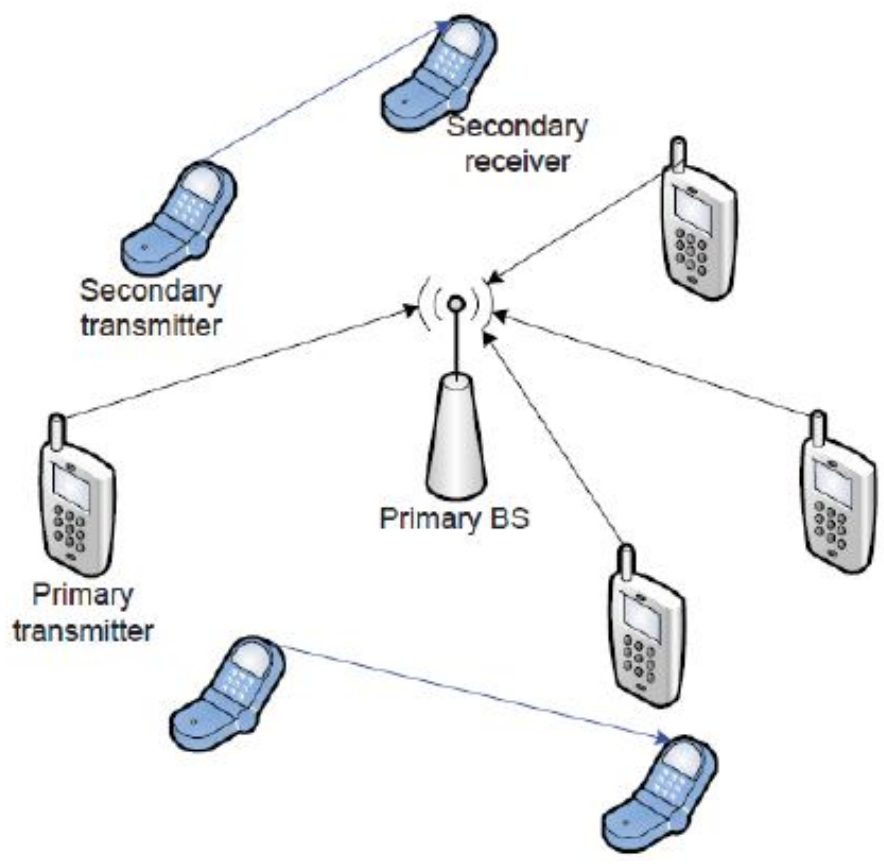

Figure 1. System model, cognitive underlay network.

The system model for underlay cooperation is shown in figure1.the primary transmitter signal is detected by the SUs. These transmit two signals, one intended for the primary receiver and a second one intended to the secondary receiver. The primary network is assumed to consist of $K$ primary users communicating with BS. We focus on communication in the uplink direction. The secondary network consists of $N$ secondary links, each secondary link comprises one transmitter and a corresponding receiver. The secondary users are assumed to communicate in an ad-hoc fashion and they are allowed to transmit such that the interference they create on the BS is below a maximum limit $I$. All receivers, primary and secondary, are assumed to treat interference as noise. The transmit power of primary user is $p_{p}^{j}, \mathrm{j}=1, \ldots \mathrm{k}$. The channel gain between primary user $j$ and the $\mathrm{BS}$ is $g^{p p}{ }_{o}$.

Hence the signal-to-interference-plus noise- ratio (SINR) for each primary link is given by,

$$
x_{j}^{p}=P G^{p} a \frac{p_{j}^{p} g_{0, j}^{p p}}{\sum_{l=1, l \neq j}^{K} p_{l}^{p} g_{0, l}^{p p}+\eta+N_{0}}, \quad j=1,2, \cdots \cdots, K,
$$

where $P G^{p}$ is the processing gain in case that primary users are employing a spread spectrum technique $N_{0}$, is the noise power at the frequency band of operation, and $n$ is the interference caused by secondary users on the primary receiver where

$$
\eta=\sum_{i=1}^{N} p_{i}^{s} g_{0, i}^{p s}
$$


the transmit power of secondary link $i$ is $\mathrm{p}_{\mathrm{i}}^{\mathrm{s}}, i=1 \cdots, N$ and $g^{p s}{ }_{o, i}$ is the channel gain from the transmitter of secondary link $i$ to the primary BS. There are two important issues about this problem. First, it may be infeasible and an admission/removal control algorithm is needed to admit the maximum possible number of links. Second, the objective function is non-convex and global optimum solution is computationally intractable. Consequently, we decompose problem into two sub-problems and provide a solution for each of them.

For the first sub-problem, in case that not all secondary links can be supported under the given constraints, admitting the maximum possible number of links to the network is an NP-hard problem as has been shown in [6]. Hence, we propose a suboptimal solution to this problem that can be implemented by secondary links in a distributed manner and achieve a close-to-optimum solution. For the second sub-problem, we tackle the non-convexity of the objective function by introducing an iterative algorithm based on successive solutions to approximate geometric programs of the original problem.

\section{ALOGRITHMS FOR MAXIMIZE THE ADMITTED LINKS}

The admission control problem has been addressed before in previous works and was first introduced for conventional networks in, then in spectrum underlay cognitive networks, where the additional constraint of the interference limit on primary users is imposed. For this case, new admission control algorithms have been developed to account for the new constraint [4]-[6]. Most of those algorithms use the distributed constrained power control (DCPC) to check the feasibility of the current set of links.

\subsection{DCPC ALGORITHM}

The DCPC algorithm is introduced in [11] and it aims at allocating power to links such that the required SINR for each secondary link is achieved at the minimum possible transmission power, provided that all links can be supported at their QoS requirements.

\subsection{DISTRIBUTED ONLINE ALGORITHM}

One of the efficient techniques for link selection is I-SMIRA proposed in [6] which is a removal algorithm that consists of two phases: a DCPC phase and a removal phase. For the removal phase a centralized removal criterion that accounts for the interference constraint is developed, where after the DCPC phase, if a constraint is being violated, the removal criterion selects a single user for permanent removal. By removing one user, the DCPC is performed by the remaining set of users and the constraints are checked so as to call the removal phase if any constraint is violated or to terminate the algorithm otherwise, where all the users performed the last DCPC phase are admitted.

\section{MAXIMIZATION OF THE SUM THROUGHPUT}

Now assume that a feasible set of secondary links are obtained by any admission control algorithm. It is required to allocate power to the admitted secondary links such that the sum throughput of all links are maximized without violating the original constraints. Without interference and QoS constraints, the global optimal solution is determined for $\mathrm{N}=2$ to be in the form of binary power allocation with each user either remaining silent or transmitting with 
maximum power. Moreover, the authors of [10] showed via numerical simulations that binary power allocation almost always achieves the global optimal solution for more than two users. It is also shown that binary power allocation achieves the global optimal solution when the system is in the interference-limited or noise-limited regimes.

The main idea behind binary power allocation is that, the optimal solution often lies on a corner point of the rate region. Those corner points are determined by binary power allocation. For the unconstrained case, the number of those corner points is $2^{\mathrm{N}}-1$ and obtaining the optimal binary power allocation solution is an exhaustive search problem with exponential complexity. For this reason, several papers addressed reduced complexity binary power allocation algorithms [10], [8]. In the constrained case where we must satisfy QoS requirements for the secondary links as well as interference constraint for the BS, binary power allocation may not achieve an efficient solution. The reason is that the corner points of the new achievable rate region are quite difficult to calculate as they are no longer characterizable by binary power allocation. Moreover, their number can be extremely large. This has led to techniques based on approximating the nonconvex problem into a sequence of geometric programs that can provide efficient suboptimal solutions.

\section{RESULTS AND DISCUSSIONS}

Figure2 shows that power control for spectrum sharing cognitive radio networks and figure 3 shows that admission control for cognitive radio networks. Here we consider 2 cases allowed users with and without box constraints. In power control figure is plotted for total user Vs transmitted power and figure 3 plotted for total user Vs admitted user.

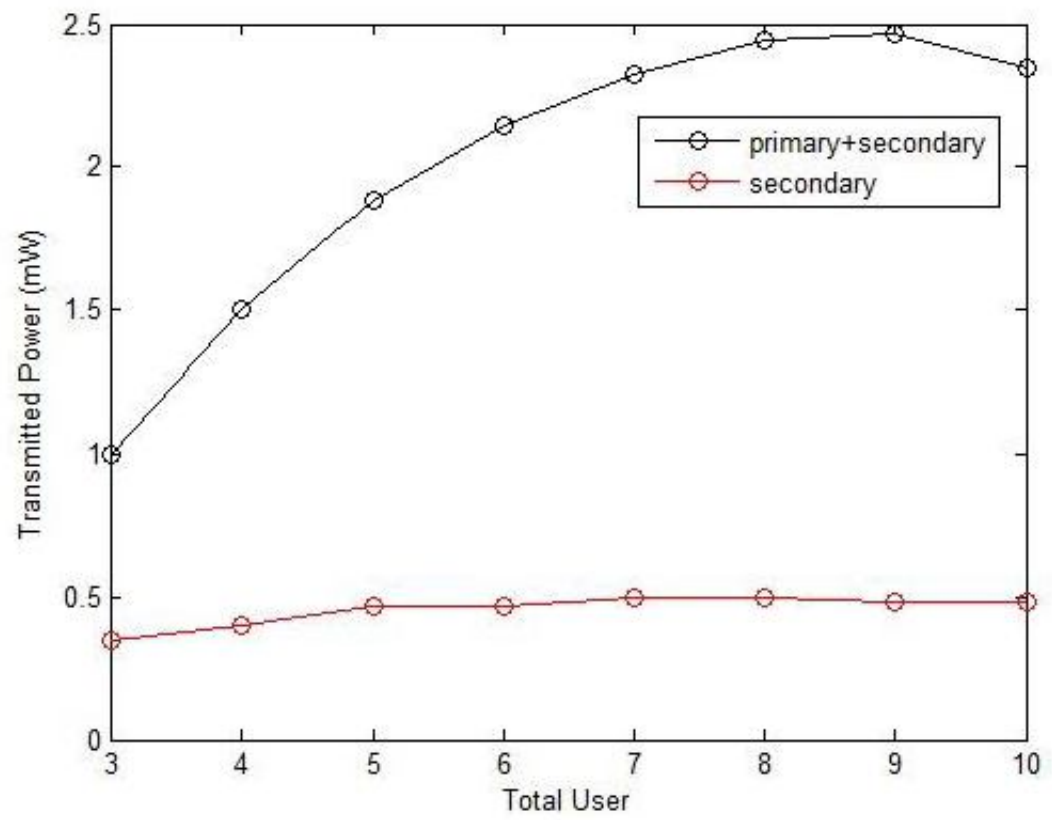

Figure 2. Power Control 
Advanced Computing: An International Journal (ACIJ), Vol.5, No.2/3, May 2014

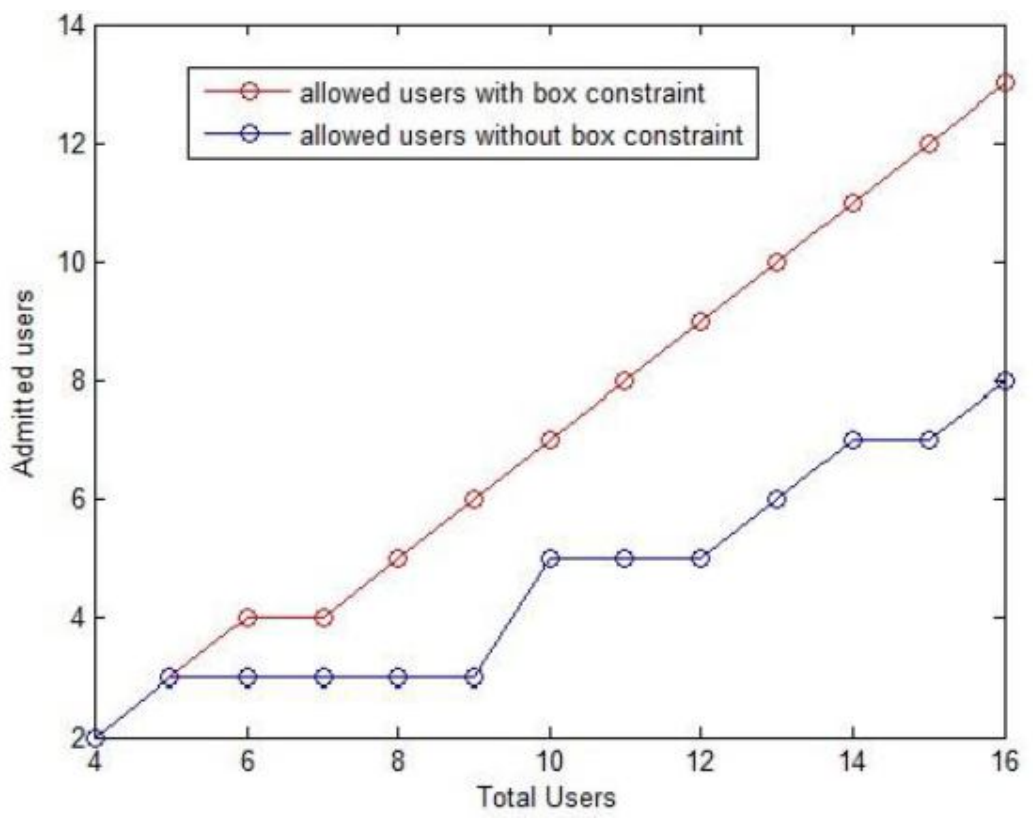

Figure 3. Admission Control

\section{5 . CONCLUSION}

Our aim in this paper has been to maximize the sum throughput for the maximum number of secondary links admitted to an underlay cognitive network under two main constraints: interference limit on the primary receiver and minimum QoS guarantees for each secondary link. We have divided the problem into two parts. In the first part we have considered the admission of maximum number of links that can be supported under the two constraints. The second part is concerned with maximizing the sum throughput of the admitted secondary links under the same constraints. Finally, we have found numerically that the QoS guarantees do not result in a significant loss in the sum throughput of the secondary network, instead, they allow more secondary links to operate reliably. In MIMO system to minimize the transmit power of the SUtransmitter while simultaneously targeting a lower-bound on the received signal-to-interferenceplus-noise-ratio(SINR) for the SU's and imposing on upper limit on the interference power(IP) at the PUs.

\section{REFERENCES}

[1] FCC, Spectrum policy task force report, FCC 02-155, Nov. 2002.

[2] FCC, Facilitating opportunities for flexible, efficient, and reliable spectrum use employing cognitive radio technologies, notice of proposed rule making and order, FCC 03-322, Dec. 2003.

[3] J. Mitola III, "Cognitive radio: an integrated agent architecture for soft-ware defined radio," Doctor of Technology dissertation, Royal Institute of Technology (KTH), Sweden, May 2000.

[4] Y. Xing, C. Mathur, M. Haleem, R. Chandramouli, and K. Subbalak-shmi, "Dynamic spectrum access with QoS and interference temperature constraints," IEEE Trans. Mobile Comput., vol. 6, no. 4, pp. 423-433, Apr. 2007.

[5] M. H. Islam, Y. C. Liang, and A. T. Hoang, "Distributed power and admission control for cognitive radio networks using antenna arrays," in Proc. IEEE DySPAN'07, pp. 250-253, 17-20, Apr. 2007. 
[6] L. Le and E. Hossain, "Resource allocation for spectrum underlay in cognitive radio networks," IEEE Trans. Wireless Commun., vol. 7, no. 12, pp. 5306-5315, Dec. 2008.

[7] D. Kim, L. Le, and E. Hossain, "Joint rate and power allocation for cognitive radios in dynamic spectrum access environment," IEEE Trans. Wireless Commun., vol. 7, pp. 5517-5527, Dec. 2008.

[8] J.Tadrous, A. Sultan, and M. Nafie, "Power control for constrained throughput maximization in spectrum shared networks," in Proc. IEEE Global Telecommun. Conf., 2010.

[9] J. Papandriopoulos and J. S. Evans, "SCALE: a low complexity distributed protocol for spectrum balancing in multiuser DSL networks," IEEE Trans. Inf. Theory, vol. 55, no. 8, pp. 3711-4843, Aug. 2009.

[10] A. Gjendemsjo, D. Gesbert, G. Oien, and S. Kiani, "Binary power control for sum rate maximization over multiple interfering links," IEEE Trans. Wireless Commun., vol. 7, no. 8, pp. 3164-3173, Aug. 2008.

[11] S. A. Grandhi and J. Zander, "Constrained power control," Wireless Personal Commun., vol. 1, no. 4, 1995.

\section{Authors}

A. Suban, received B.E in the department of Electronics and Communication Engineering from Anna university, Chennai and M.E in the discipline of Wireless Technology from Thiagarajar College of Engineering, Madurai in 2011. He is currently working as Assistant Professor in the Department of Electronics and Communication Engineering, Velammal College of Engineering and Technology, Madurai-625009, Tamil Nadu, India. His area of interest in Signal processing mainly focused on MIMO techniques with beamforming, OFDM and power control techniques.

I.Ramanathan, received his B.E degree National Engineering college, Kovilpatti, Anna University, Tirunelveli, Tamil Nadu. He is doing M.E (Communication Systems) at Velammal College of Engineering and Technology, Madurai, Tamil Nadu, India. He has published 2 Technical papers International Conferences and National Conferences.
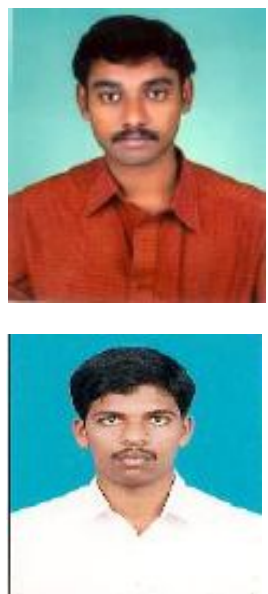